\title{
Scalloped hammerhead sharks have more complex migratory routes than expected
}

Using a new method to scalloped hammerhead sharks, Felipe Coiraton and Dr the National Autonem a University of Mexico, identified a more complex picture than that not all sharks follow the same pattern of movements, with some preferring to stay opt for deeper waters. This appropriate conservation appropriate conservation endangered species. study migratory routes of previously thought. It turns out in coastal areas while others
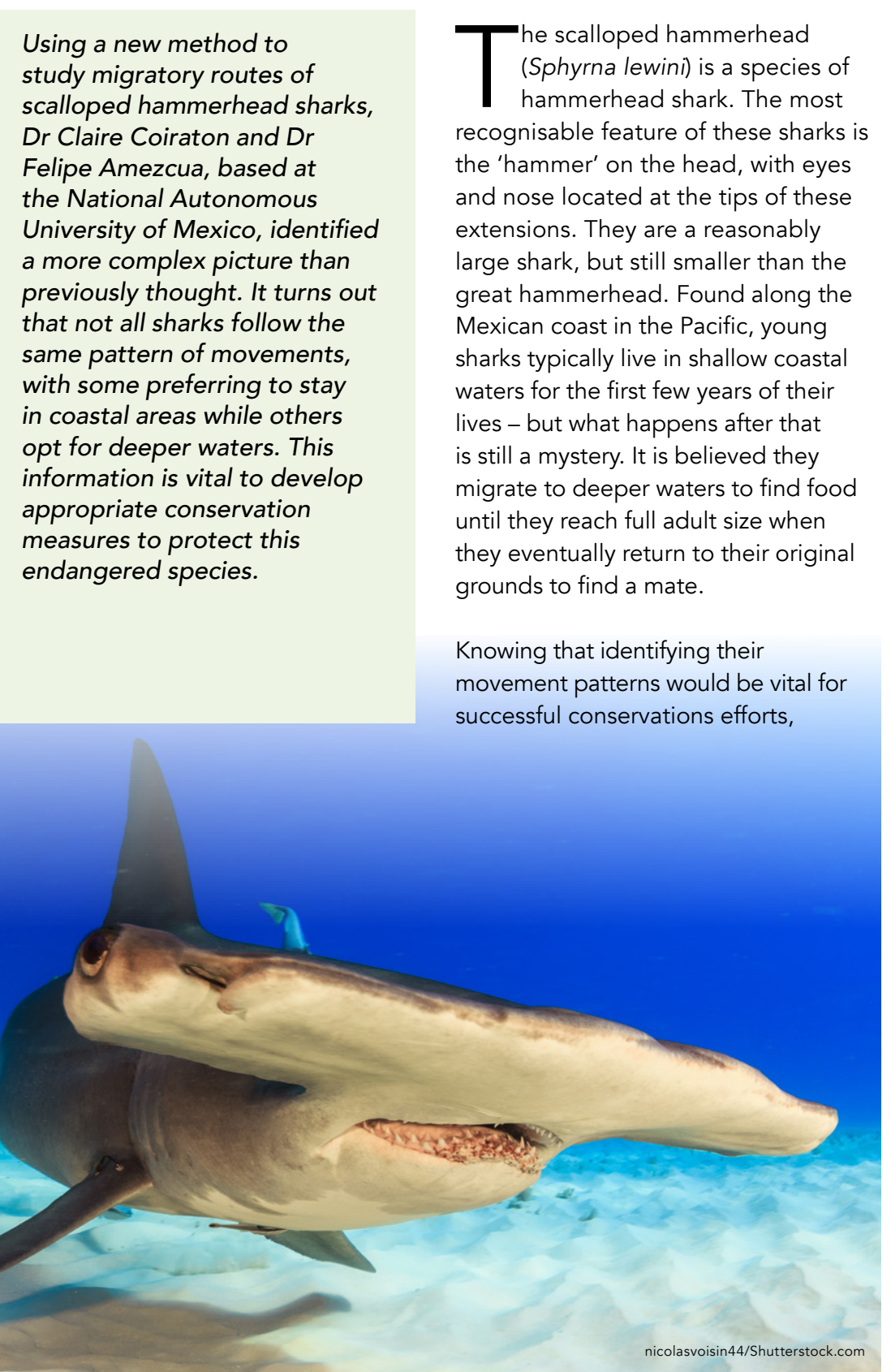

Dr Claire Coiraton and Dr Felipe Amezcua, both based at the National to shed some light onto these migrat routes. Physical tagging is undoubtedly the most common way to study animal migrations, but the researchers are keen to try a different approach. Their method of using in-utero vertebral microchemistry has not been tested on sharks before, but Dr Coiraton and Dr Amezcua believe it has great potential to use with these animals.

\section{MICROCHEMICAL SIGNATURES} The idea is simple: analyse the chemical composition of the sharks' vertebrae, which should be a reflection of the composition of the water where a powerful tool to assess the place poirtu tool to assess the place several marine species. "Recent work suggested that chemical signatures derived from trace elements deposited in the vertebrae of sharks during
growth reflect the environmental conditions of the water mass in which they have been exposed", explain Dr Coiraton and Dr Amezcua.

In other words, certain chemical elements present in the water are continuously incorporated into the vertebrae as sharks grow. They serve as markers to pinpoint the sharks'
movements. For the researchers, "this movements. For the researchers,
offers great potential to examine offers great potential to examine
questions related to habitat and questions related to habitat and
migration patterns of hammerhead
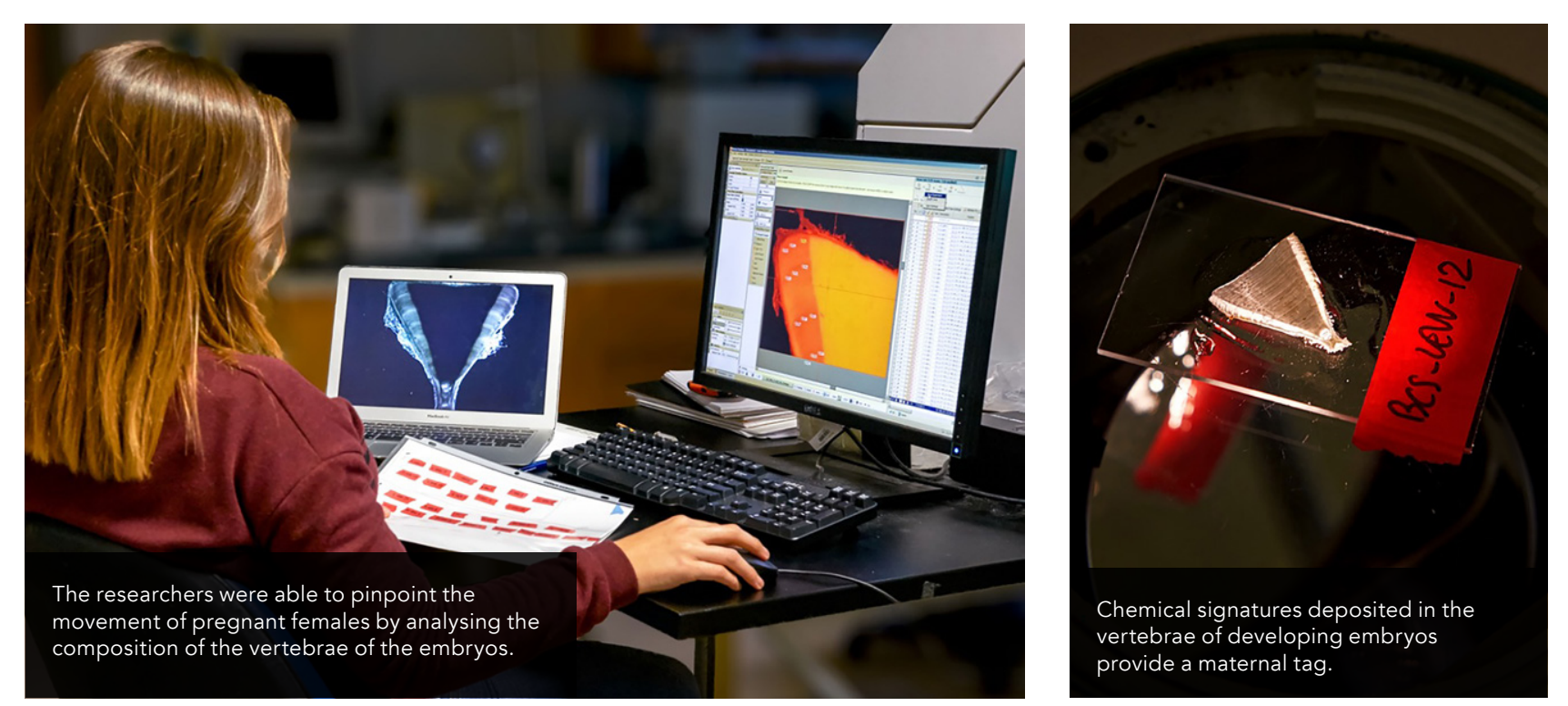

sharks by analysing the vertebra elemental signatures".

Three chemical elements are key for this pproach: strontium (Sr), barium (Ba) and lead (Pb). The first two are typically analysed as a ratio to indicate the level of salinity: strontium is abundant in deep water with high salinity, whereas barium is present in regions with low salinity. Levels of lead complement this information by highlighting polluted
areas, usually associated with more coastal environments.

\section{MATERNAL TAC}

To establish the viability of using this new approach, Dr Coiraton and Dr Amezcua started by testing vertebrae
from pregnant females and their embryos. Scalloped

embryos. Scalloped

gestation period of 10 e quite a long giving plenty of time for the months, supply of nutrients to influence the composition of vertebrae of the embryos while in utero. This mechanism is possible only atter the fomation of the placen when shark embryos are nourished directly from the mother's bloodstream.

With the new approach, Dr Coiraton and Dr Amezcua were not only able to identify embryos from the same litter, but they could also match each litter to their respective pregnant female. "As a vivparous species with signatures deposited in the vertebrae of developing embryos provide a maternal their mother" explain the researchers.

\section{MIGRATORY PATTERNS}

\section{FOR FEMALES}

Once they established that the new chemical approach was a viable method to study migratory patterns of hammerhead sharks, the team could Their results con out their travels. females and males tend to stay in coastal waters for the first few years of their lives. Surprisingly, females opt to leave hese waters at a younger

t turns out that hammerhead shark exhibit more variable migratory patterns than expected.

age than males: $3-5$ years in females compared to 4-7 in males. Researcs believe females go out searching for high-energy foods sooner than males to obtain the larger body size needed for future pregnancies.

After about a decade, females return to the same location during gestation. Curiously, vertebrae analyses detected two distinct migratory patterns for pregnant females. Most females stay offshore during the initial stages of gestation only to decide to quickly bith, whereas the remaning fero birch, whereas he remaining females in gestation and stay in the same declining as they moved from deep to coastal waters, while the second group showed more constant ratio indicative of more restricted movements.

"The reasons why some females presumably remained nearshore during the gestation are unknown", say Dr Coiraton and Dr Amezcua, "especially considering that adults ought to migrate offshore as a their foraging press by feeding on energy-rich groups of pregnant females in different locations can minimise the risk of poor environmental poor environmen
conditions and

maximise the chances of tar pregnancy to full term.

This phenomenon is known as partial migration and is considered a strategy to limit predator attacks or unfavourable environmental conditions. Even if one group of pregnant females falled to reach full term due to low food levls, the other group would still be severe dosves their young and prevent (a)

However, there are still many unanswered questions. Not all some anima living panty diff 


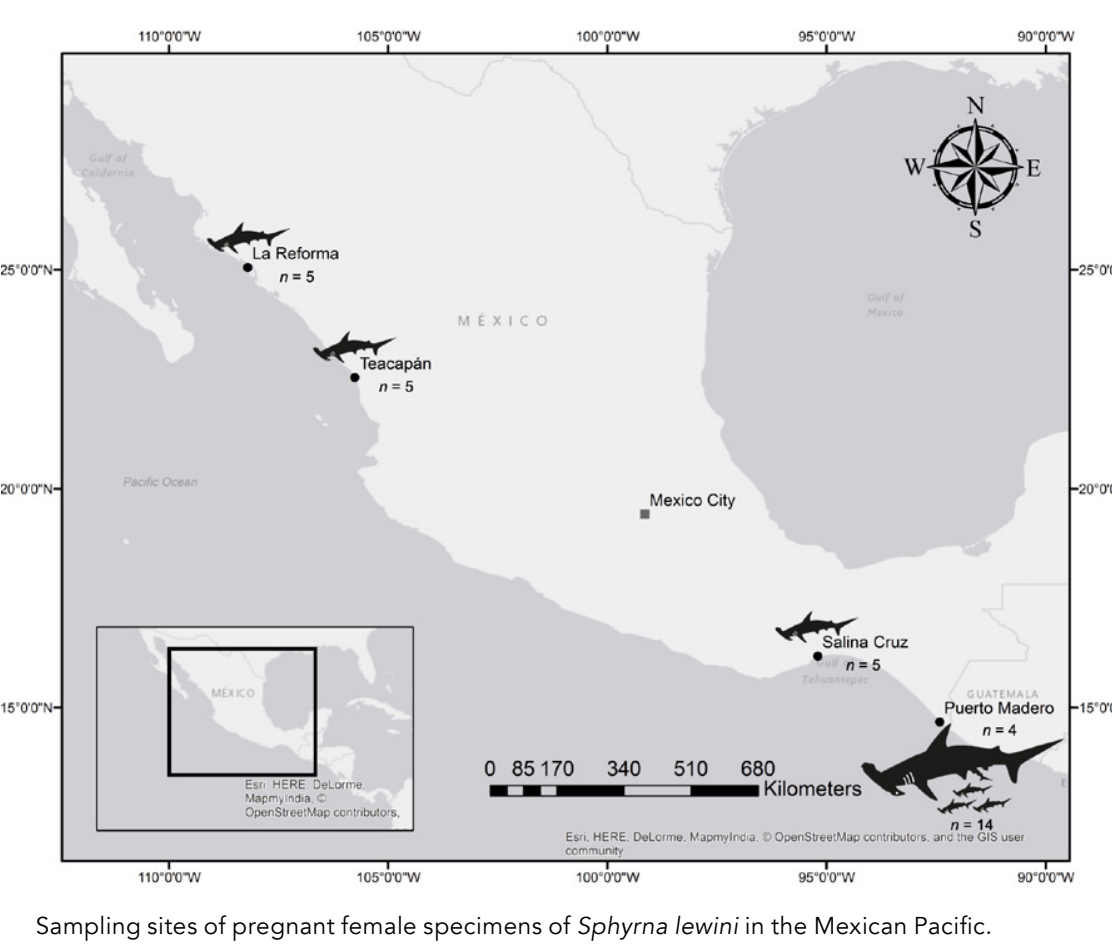

The migratory patterns unveiled by $\mathrm{Dr}$ Coiraton's and Dr Amezcua's team are going to be essential to establish new conservation strategies to protect this allreadly endangered species.

habitats during their lifetime. Also, it The reason why some males return is important to note that this approach to the shore or never leave in the first only detects movements that last at place is still unexplained, as they do least three weeks. Any short trips where need to stay in shallow waters. The animals leave that location quickly is not enough to leave a mark that can be

\section{MIGRATORY PATTERNS FOR MALES} When it comes to males, results also movements. After staying near the shore for the first few years of their lives, some sharks eventually migrate to deeper water. For some males, this is a decision that lasts a lifetime, while others return to coastal waters after living offshore for a period. Curiously there is also a third group of mal sharks that remain nearshore all their lives. The research team believes th alternating among these strategies might have important impacts on the fishing of the population to the individuass might be me reproductive to fisheries mile remaining neeptible

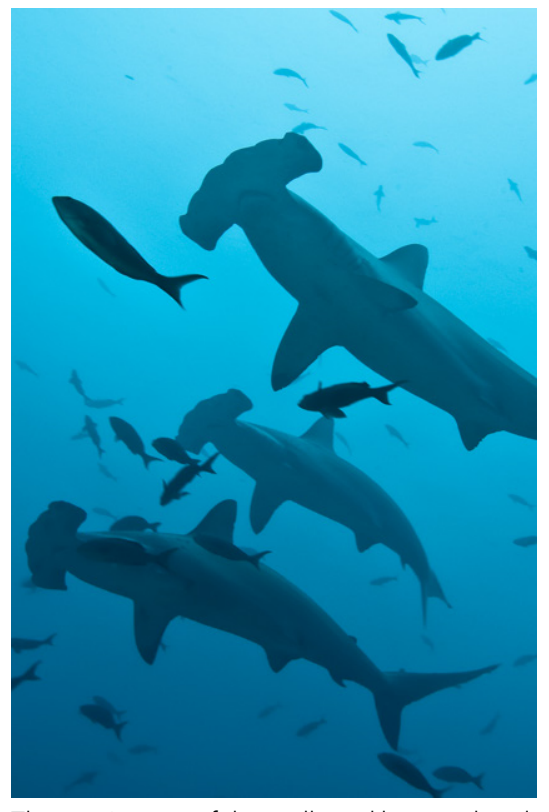

The persistence of the scalloped hammerh
shark in the Mexican Pacific, despite heavily shark in the Mexican Pacific, despite hear
fished, might be due to its habitat use. researchers suggested that these males may be lookng for an opportunity to coast to give birth.

It turns out that hammerhead sharks exhibit more variable migrato patterns than expected. They seen to have a stronger affinity to shallow and coastal waters, with only a limited number of animals opting for deepwater habitats for their entire life. Most males will at some point return to coastal waters.

\section{CONSERVATION EFFORTS}

Sadly, most species of hammerhead sharks have disappeared from Mexico's Pacific Coast. Nevertheless, is still preval hammerhead shark bevaris fished. According to Dr Coiraton and Dr Amezcua, the persistence of scalloped hammerhed sharks in the Mexican Pacific "might be linked to its behavioural plasticity of movement and habitat use rather than its life history characteristics (i.e. slow growth, late age at maturity)". In other words, the fact that not all animals follow the same migratory pattern seems to be an advantage, allowing them to survive where similar species have failed.

However, this makes studying this species and understanding their movements even more complex. For the authors, assessing their migratory routes is the first step to "ensure hammerhead shark in the Mexican Pacific are properly protected through conservation and management tools".

Considering how long some animals stay in coastal waters, current fishing measures, such as seasonal prohibition between May and July only, may not be adequate to ensure sustainable management of the shark population Pregnant females, in particular, are when they return nearshore during gestation. The migratory patterns unveiled by Dr Coiraton's and Dr Amezcua's team are going to be essential to establish new conservation strategies to protect this already endangered species.

\section{Behind the Research}

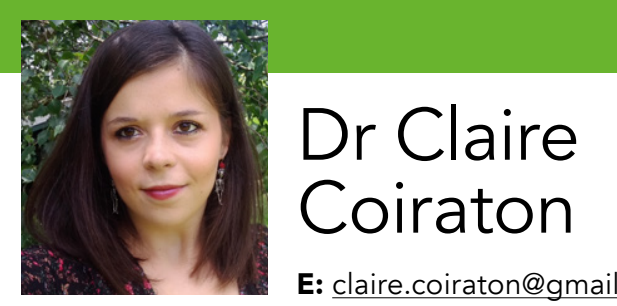
T: $+5216692134016 \mathbf{W}$ : $\mathrm{https://www.}$
researchgate.net/profile/Claire_Coiraton

Research Objectives

$\mathrm{Dr}$ Coiraton and Dr Amezcua examine the vertebra hammerhead shark to infer the species' life-tim migration patterns.

\section{Detail}

Instituto de Ciencias del Mar y Limnología Universidad Nacional Autónoma de 1 Mazatlán 82040, Sinaloa, Mexico

Dr Claire Coiraton is recently graduated with a PhD in marine biology from the National Autonomous are the spatial ecology and behavioural plasticity of elasmobranchs to evaluate how interactions between life history traits and ecology may affect a population response to fisheries and environmental changes and achieve sustainable fisheries practice.

Dr Felipe Amezcua is a fish biologist and professor at the Institute of Ocean Sciences and Limnology of the National Autonomous University of Mexico. His ecology of estuarine and coastal fish species, mangrove food webs and productivity, fish contamination by trace change on marine ecosystems.

Funding

Funding for this research was provided by CONACYT, the project PAPIIT-IG201215, the Western Division of

\section{Collaborators}

Artisanal fishers from Puerto Madero, Teacapán, Mazatlán and La Reforma

and th. Peebles and David L. Jones at the University of South Slorida St. Petersburg, Florida, USA Allan Valencia, Michey Cervantes and Eliazar Armenta Valenzuela - Hans J. Hartmann

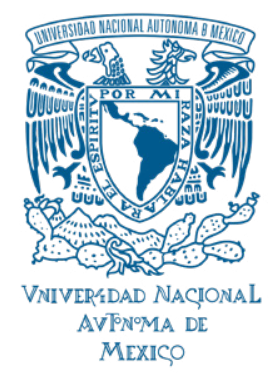

\section{6e). Dr Felipe IS Amezcua \\ E: famezcua@ola.icmyl.unam.mx} T: +5216699852845 W: https://www.
esearchgate.net/profile/Felipe_Amezcua

\section{References}

Coiraton, C. and Amezcua, F. (2020). In utero elemental tags lewini reveal migration patterns of pregnant females. Scientific Reports, 10, 1799. Available at: https://doi.org/10.1038/ s41598-020-58735-8

Coiraton, C., Amezcua, F., and Ketchum, J. (2020). New insights into the migration patterns of the scalloped microchemistryark Sphing riolowin 167, 58. Available at: https:// microchemistry. Marine Biology, 167,
doi.org/10.1007/s00227-020-3668-0

\section{Personal Response}

How could this work help in the development otect the

II Migration is vital for marine species adaptability and the exchange of individuals among geographically separate groups, as well as the resilience of a population to harvest. Identifying patterns of is therefore crucial to ensure that populations are
properly protected through conservation and fishery properly protected
management tools.

Considering that scalloped hammerheads exhibit stronger affiliation with coastal habitats than previously assumed, and that most pregnant females returned to coastal habitats before term, better management extension of the currently implemented fishery closure (May-July) to protect those females entering sooner th nearshore habitats (around March), as well as most of the neonates before these begin moving away from the shore around late August. These measures should help preserving the reproductive potential of this critically

The establishment of a minimum catch size does not seem an adequate solution for this species as it is fusceptible to be incidentally caught anyway by other anatomy of its head and the diversity of fishing gea used in the region. 